\title{
Maternal uniparental disomy 7 in Silver-Russell syndrome
}

\author{
M A Preece, S M Price, V Davies, L Clough, P Stanier, R C Trembath, G E Moore
}

\begin{abstract}
Silver-Russell syndrome (SRS) is characterised by intrauterine and postnatal growth failure accompanied by a variable number of dysmorphic features. It is usually sporadic although a few familial cases have been described. In a prospective study of 33 patients with sporadic SRS, we have studied the parent of origin of chromosome 7 using variable number tandem repeat (VNTR) or microsatellite repeat markers and have identified two patients with maternal uniparental disomy of chromosome 7 (mUPD7). In one family, inconsistent inheritance of paternal alleles of markers on chromosomes other than 7 led to their exclusion from further study. The probands were clinically mild and symmetrical, but showed no gross clinical differences from the $\mathbf{3 0}$ patients with chromosome 7 derived from both parents. (f Med Genet 1997;34:6-9)
\end{abstract}

Keywords: Silver-Russell syndrome; maternal uniparental disomy; chromosome 7; intrauterine growth retardation.

Institute of Child

London, 30 Guilford

Street, London

WC1N 1EH, UK

M A Preece

Action Research Laboratory for the

Molecular Biology of

Fetal Development,

Institute of Obstetrics

and Gynaecology,

Royal Postgraduate

Medical School,

Queen Charlotte's and

Chelsea Hospital,

Goldhawk Road,

London W6 OXG, UK

M A Preece

V Davies

P Stanier

G E Moore

Department of

Clinical Genetics,

Leicester Royal

Infirmary, Leicester

LE1 5WW, UK

$S M$ Price

Department of

Medicine and

Therapeutics and

Genetics, University of

Leicester, Leicester

LE2 7RH, UK

L Clough

R C Trembath

Correspondence to: Professor Preece.

Received 8 July 1996 Revised version accepted for publication

1 August 1996

are intrauterine and postnatal growth retardation, relative sparing of cranial growth, triangular face, downturned corners of the mouth, and, in a large proportion, clinodactyly and facial, limb, or truncal asymmetry. The postnatal growth pattern is characteristic with persistent short stature, marked leanness, and a tendency for early, or at least prompt, puberty with further restriction of adult height. ${ }^{34}$ More recently a late appearing cognitive deficit has been described in approximately $50 \%$ of 25 patients. $^{5}$

In 1995, Kotzot et al reported maternal uniparental disomy 7 (mUPD7) in three patients with SRS from a sample of 25 clinically identified cases and in one case from 10 patients with primordial dwarfism (PD). The distinction between SRS and PD was that PD had the same growth characteristics but no dysmorphic features. Complete maternal isodisomy was found in two of the SRS patients and the one with PD, whereas one SRS child showed maternal heterodisomy. In addition, there have been four case reports of mUPD $7^{7-10}$ associated with low birth weight and poor postnatal growth. In each of the first two ${ }^{78} \mathrm{mUPD} 7$ was found in a patient with cystic fibrosis where carrier status was confined to the mother. Both patients showed pre- and postnatal growth re- tardation and one $\mathrm{e}^{7}$ had some features of SRS The third case showed maternal isodisomy ascertained during investigation of the COL1A2 locus for which he was homozygous. ${ }^{9}$ The proband was of low birth weight for gestational age and was very short at 4 years and as an adult; he was said to have a small, pointed face. A further case with pre- and postnatal growth retardation associated with maternal uniparental heterodisomy has recently been reported ${ }^{10}$; there were no dysmorphic features suggestive of SRS. Finally, a single case has been reported of mUPD7q and paternal UPD7p in a child who was of normal birth weight but who grew poorly after birth, being significantly short at 2 years ${ }^{11}$; some minor dysmorphic features compatible with SRS were noted.

One drawback of all the the above studies (including those of Kotzot $e t a l^{6}$ ) is that the clinical features of the patients were assessed by a number of different clinicians. We now report our molecular genetic studies in 33 SRS patients, all of whom were clinically assessed by a single clinical geneticist according to preset diagnostic criteria. Following clinical appraisal, full details of which are to be published elsewhere, parent of origin of each proband's chromosome 7 was determined using a combination of Southern analysis with VNTR markers and the polymerase chain reaction (PCR) with microsatellite repeat markers.

\section{Materials and methods}

Thirty three probands were ascertained from clinical colleagues and they and their families visited by one of us (SMP) and clinically assessed according to the following criteria: low birth weight ( $>2$ SD below mean), short stature at the time of our assessment $(>2$ SD below mean), characteristic facial features, and facial, trunk, or limb asymmetry. Body measurement data were converted to standard deviation scores using British reference data. ${ }^{12}$

High resolution cytogenetic examinations were performed in all probands using blood lymphocyte cultures.

DNA was extracted by conventional methods. Both parents and the proband were studied using Southern blot analysis with two VNTRs and PCR with 16 microsatellite repeat markers distributed along the length of chromosome 7. For both VNTRs, genomic DNA was restricted with HinfI followed by blotting and hybridisation using standard methods. Microsatellite repeats were analysed using fluorescently labelled primers by standard semiautomated methods as previously 

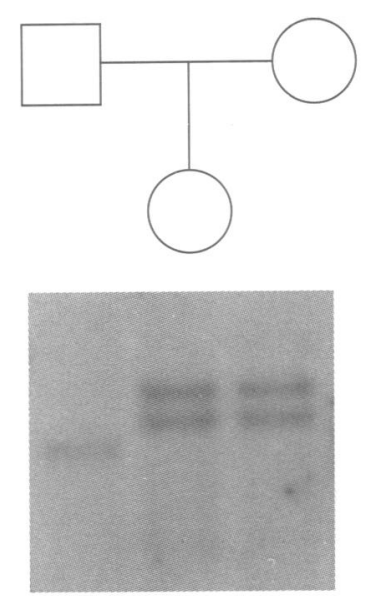

B
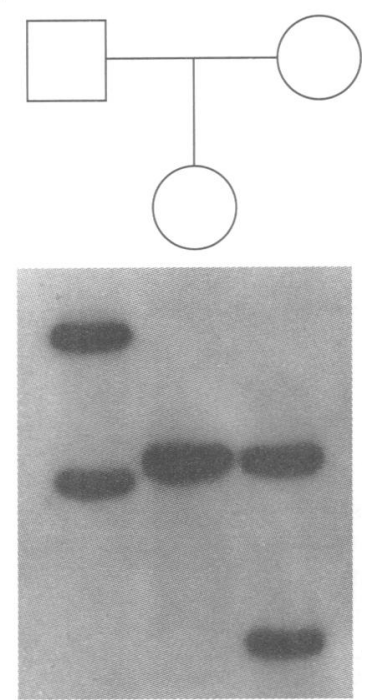

Figure 1 Autoradiograph of Southern blot for family 1 $(A)$ and family 2 (B) after DNA was restricted with HinfI and hybridised with D7S22.

described. ${ }^{13}$ VNTRs and microsatellite repeat markers on chromosomes $2,6,11$, and 17 were studied to confirm paternity.

The study was approved by the Joint $\mathrm{Re}$ search Ethics Committee of Great Ormond

Table 1 Genotypes of patients 1 and 2 as determined from two VNTR probes (D7S21) and (D7S22) and 16 microsatellite repeat markers. Markers informative for parental origin are in bold. The order of alleles is father/proband/mother; the column to the right of each patient's data indicates regions where the markers indicate isodisomy (open), heterodisomy (filled), or were not informative in this respect (grey)

\begin{tabular}{|c|c|c|c|c|c|}
\hline Marker & Location & Family 1 & & Family 2 & \\
\hline $\begin{array}{l}\text { D7S21 } \\
\text { D7S531 } \\
\text { D7S513 } \\
\text { D7S493 } \\
\text { D7S629 } \\
\text { D7S484 } \\
\text { D7S519 } \\
\text { D7S502 } \\
\text { D7S669 } \\
\text { D7S524 } \\
\text { D7S527 } \\
\text { D7S486 } \\
\text { CFTR } \\
\text { D7S530 } \\
\text { D7S684 } \\
\text { D7S483 } \\
\text { D7S550 } \\
\text { D7S22 }\end{array}$ & $\begin{array}{l}\text { 7pter-p22 } \\
\text { 7pter-p22 } \\
\text { 7p21-p13 } \\
\text { 7p21-p13 } \\
\text { 7p21-p13 } \\
\text { 7p211-p13 } \\
\text { 7p113-p12 } \\
\text { 7p11.2-p11.1 } \\
\text { 7q11.23-q21.1 } \\
\text { 7q11.23-q21.1 } \\
\text { 7q21.3 } \\
\text { 7q31.1-q31.3 } \\
\text { 7q31.3 } \\
\text { 7q32-q34 } \\
\text { 7q32-q34 } \\
\text { 7q35-qter } \\
\text { 7q35-qter } \\
\text { 7q36-qter }\end{array}$ & 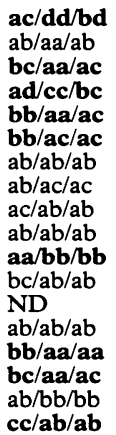 & $\begin{array}{l}\square \\
\square \\
\square \\
\square \\
\mathbf{6} \\
\mathbf{8} \\
\mathbf{8} \\
\mathbf{8} \\
\mathbf{0} \\
\square \\
\square\end{array}$ & 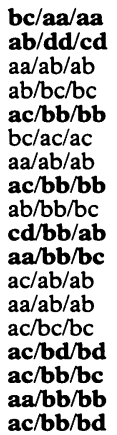 & 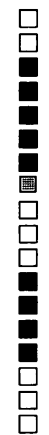 \\
\hline
\end{tabular}

Street Hospital and the Institute of Child Health, approval number 1278.

\section{Results}

THE STUDY POPULATION

There were 33 probands with both parents available for study, 17 males and 16 females, aged between 10 months and 35 years at the time of examination. Twenty nine subjects had a birth weight $>2$ SD below the mean and the remaining four term babies had birth weights between 2500 and $2700 \mathrm{~g}$. Classical facial features, as described by Russell, ${ }^{2}$ were seen in 23 , while 10 had a milder facial phenotype. Limb asymmetry of $\geq 1.0 \mathrm{~cm}$ associated with facial asymmetry was present in 12 subjects. At the time of birth of the probands, maternal age range was 17.8 to 34 years and paternal age range 18.8 to 35 years. All had a normal karyotype.

PATERNITY

Analysis of VNTRs and microsatellite markers on chromosomes $2,6,11$, and 17 was consistent with paternity as stated in 32 families. In one family, with a female proband, inconsistent inheritance of paternal alleles at four markers led to their exclusion from further analysis.

MARKERS OF CHROMOSOME 7

In two families, marker analysis showed mUPD7. Both VNTR markers were informative in both families. For family 1, D7S21 showed isodisomy, and for family 2 both mother and child were homozygous for alleles different from the father. In the case of D7S22, proband 1 was heterodisomic whereas proband 2 showed isodisomy for one maternal allele (fig 1A, B).

In family 1 , there were an additional seven informative microsatellite markers and in family 2, eight informative microsatellite markers (table 1). Cytogenetic localisation and order of all the markers were taken from Tsui et al. ${ }^{14}$

In both patients, all markers informative for parent of origin showed mUPD7. Considering only the proband and maternal markers, it was possible to explore the nature of the disomy, that is, whether the proband had inherited a copy of each of the mother's chromosome 7 (heterodisomy) or two copies of one chromosome (isodisomy). In family 1 , six markers indicated definite isodisomy, eight markers definite heterodisomy, and three were not informative as the mother was homozygous. In family 2 , six markers indicated definite isodisomy, eight markers definite heterodisomy, and four were not informative. In this analysis, the simplest model, based on the least number of recombinations compatible with the data, was assumed.

PATIENTS WITH MUPD7

The clinical features of the two patients with mUPD7 are shown in table 2 . They showed no major differences from the other 30 patients, 
Table 2 Principle clinical features and family details of the two probands with mUPD7. The body measurements are followed by their standard deviation scores

\begin{tabular}{|c|c|c|}
\hline & Proband 1 & Proband 2 \\
\hline $\begin{array}{l}\text { Gender } \\
\text { Age of mother at birth (y) } \\
\text { Age of father at birth (y) } \\
\text { Birth weight }(\mathrm{g}) \\
\text { Gestation (wk) } \\
\text { Age at examination (y) } \\
\text { Height at examination (cm) } \\
\text { Weight at examination }(\mathrm{kg}) \\
\text { Head circumference (cm) }\end{array}$ & $\begin{array}{l}\text { Female } \\
34 \\
35 \\
2010 \quad(-2.49) \\
38 \\
4.5 \\
93(-2.57) \\
12.1(-2.91) \\
50.5(-0.58)\end{array}$ & $\begin{array}{cc}\text { Female } & \\
29 & \\
30 & \\
2050 & (-3.22) \\
40 & \\
0.84 & \\
61 & (-4.62) \\
4.42 & (-7.37) \\
43.2 & (-2.18)\end{array}$ \\
\hline
\end{tabular}

although they were probably at the milder end of the clinical spectrum. Neither were asymmetrical. The maternal ages were not different from the remainder of the cohort (mother 1 , 34 years; mother 2, 29 years).

\section{Discussion}

In both cases reported here, the probands' and maternal alleles shown in table 1 indicate mixed disomy with a combination of iso- and heterodisomy. This is compatible with meiosis I non-disjunction with three recombinations in patient 1 and four in patient 2 . In the case of family 1 , this interpretation is strongly supported by the heterozygosity of all the markers around the centromere. There is more doubt concerning family 2 ; the proximal $7 q$ markers show isodisomy and the most proximal $7 \mathrm{p}$ marker is not informative (table 1). Therefore we cannot exclude meiosis II non-disjunction in this family. Our data lend further support to the association of mUPD7 with SRS, increasing the number of published cases with full mUPD7 and the growth characteristics of SRS to 10 , although in three of the earlier reports the abnormal growth was not associated with the more specific dysmorphic SRS features. The significance of the case of Eggerding $e t$ al ${ }^{11}$ where the mUPD only involved 7q is unclear; it is noteworthy that the birth weight was normal ( $3040 \mathrm{~g}$ at term), which is at variance with all the other reports where pre- and postnatal growth failure were a feature. Possibly the prenatal features are dependent on a gene or genes on $7 p$, whereas the postnatal features are associated with genes on $7 \mathrm{q}$.

Kotzot et al described three patients with SRS and mUPD7 where two were isodisomic and one heterodisomic. The clinical differentiation between these cases and their cases of $P D$ is of uncertain significance, particularly as one of the latter also had mUPD7. The authors comment on this and allow that the clinical diagnosis of the disomic PD case was in part subjective and the differentiation between $\mathrm{PD}$ and SRS, in this case at least, uncertain. In all four of their disomic patients their data suggest that whether chromosome 7 was isoor heterodisomic, it was complete for each individual chromosome.

The accumulated evidence supports the hypothesis that there are one or more imprinted genes on chromosome 7 important in pre- and postnatal growth. In isodisomy it is possible that part of the phenotype is a consequence of a recessive maternal gene. There is one case of $\mathrm{SRS}^{6}$ and one of pre- and postnatal growth failure ${ }^{10}$ with apparently pure heterodisomy, although in the latter case there were only eight informative markers along the length of the chromosome and a double recombination between one or more pairs of markers cannot be ruled out. In the two cases presented here there is mixed disomy, but between the two cases there is minimal overlap in the regions isodisomic for chromosome 7 . These are restricted to 7pter-p22 and 7q35-qter (table 1). Where complete heterodisomy is present, exposure of a maternal recessive gene cannot explain the phenotype and the best explanation is the presence of an imprinted gene whose expression is diminished or absent when only maternal alleles are present. The explanation in the remainder of cases of SRS may be a mutation/ deletion in the allele of paternal origin or that the condition is heterogeneous with more than one aetiology.

The connection with imprinted genes is strengthened by the fact that human chromosome 7 has homology with multiple mouse chromosome regions; of the six mouse chromosomes that are known to possess imprinted regions, four have regions of homology with human chromosome 7 (mouse chromosomes 2, 6, 11, and 12). ${ }^{15}$ Proximal mouse chromosome 11 is particularly interesting as maternal disomy of this region is associated with reduced prenatal growth. The homologous region on human chromosome 7 contains the genes for insulin-like growth factor binding proteins- 1 and -3 and for the epidermal growth factor receptor. All of these could play a part in growth regulation.

A common feature of SRS is facial, truncal, or limb asymmetry suggesting regional growth differences which could be caused by mosaicism. It is most likely that in our two cases the mUPD7 arose from a non-disjunction and the production of a trisomic line during meiosis 1 after recombination. This was then followed by trisomic rescue producing a mixed disomic line in the embryo. Mosaicism would have been apparent from the Southern analysis and in the case of the microsatellites if there were $5-10 \%$ or more of the normal cell line. ${ }^{16}$ This was not seen. However only lymphocyte DNA was analysed and it is still possible that other tissues may have mosaic lines. Both of our cases were symmetrical, but the cases of Kotzot et at were all asymmetrical and mosaicism may have played a part. As the more quantitative Southern analysis was not carried out in this study mosaicism cannot be ruled out.

In conclusion we have identified two further cases of SRS with mUPD7, but unlike earlier cases the disomy was mixed in both cases. The next stage is the identification of genes on chromosome 7, for example IGFBP1 and IGFBP3, which, if imprinted, could be associated with the SRS phenotype.

\section{Addendum}

Since this paper was submitted to the journal, a further five families with an SRS child have been studied with the same VNTRs as above and four microsatellite markers. One proband 
shows mUPD7 with both VNTRs and two of the microsatellites are informative. Other markers confirm correct paternity. As in the earlier families, the disomy is mixed with at least two regions of isodisomy and one of heterodisomy, which includes the centromere and is therefore compatible with meiosis I nondisjunction as the underlying aetiology. This makes a total of three cases of mUPD7 out of 37 families studied.

SP is grateful for support from the Child Growth Foundation and Serono Laboratories. The laboratory expenses were funded in part by a grant to MAP from the European Society for Pediatric Endocrinology Sabbatical Leave Program. We are grateful to Mr L Butler, Director of the NE Thames Regional Cytogenetics Unit for the karyotype analyses and to our clinical colleagues who referred families to the study.

1 Silver HK, Kiyasu W, George J, Deamer WC. Syndrome of congenital hemihypertrophy, shortness of stature, an elevated urinary gonadotropins. Pediatrics 1953;12:36876.

2 Russell A. A syndrome of intrauterine dwarfism recognizable at birth with craniofacial dysostosis, disproportionate short arms and other anomalies. Proc $R$ Soc Med 1954;47: 1040-4.

3 Tanner JM, Lejarraga $\mathrm{H}$, Cameron $\mathrm{N}$. The natural history of the Silver-Russell syndrome: a longitudinal study of thirty-nine cases. Pediatr Res 1975;9:611-23.

4 Davies PSW, Valley R, Preece MA. Adolescent growth and pubertal progression in the Silver-Russell syndrome. Arch Dis Child 1988;63:130-5.

5 Lai KY, Skuse D, Stanhope R, Hindmarsh P. Cognitive abilities associated with the Silver-Russell syndrome. Arch Dis Child 1994;71:490-6.

6 Kotzot D, Schmitt S, Bernasconi F, et al. Uniparental disomy 7 in Silver-Russell syndrome and primordial growth retardation. Hum Mol Genet 1995;4:583-7.

7 Spence JE, Persiaccante RG, Greig GM, et al. Uniparental disomy as a mechanism for human genetic disease. $A m \mathfrak{f}$ Hum Genet 1995;42:217-26.

8 Voss D, Ben-Simon E, Avital A, et al. Isodisomy of chromosome 7 in a patient with cystic fibrosis: could uniparental disomy be common in humans? Am f Hum Genet 1989;45:373-80.

9 Spotila LD, Sereda L, Prockop DJ. Partial isodisomy for maternal chromosome 7 and short stature in an individual with a mutation at the COLIA2 locus. Am 7 Hum Genet 1992;51:1396-405.

10 Langlois S, Yong SL, Wilson RD, Kwong LC, Kalousek DK. Prenatal and postnatal growth failure associated with DK. Prenatal and postnatal growth failure associated with
maternal heterodisomy for chromosome $7.7 \mathrm{Med}$ Genet 1995;32:871-5.

11 Eggerding FA, Schonberg SA, Chehab FF, Norton ME Cox VA, Epstein CJ. Uniparental disomy for paternal 7p and maternal $7 \mathrm{q}$ in a child with growth retardation. $A m$ f Hum Genet 1996;55:253-65.

12 Freeman JV, Cole TJ, Chinn S, Jones PRM, White EM, Preece MA. Cross-sectional stature and weight reference curves for the UK, 1990. Arch Dis Child 1995;73:17-24.

13 Coyle B, Coffey R, Armour JAL, et al. Pendred syndrome (goitre and sensorineural hearing loss) maps to chromosome 7 in the region containing the nonsyndromic deafness gene DFNB4. Nat Genet 1996;12:421.

14 Tsui LC, Donis-Keller H, Grzeschik KH. Report of the second international workshop on human chromosome 7 mapping, 1994. Cytogenet Cell Genet 1995;71:2-18.

15 Beechey CV, Cattanach BM. Genetic imprinting map. Mouse Genome 1995;93:89-92.

16 Pangalos C, Avramopoulos D, Blouin JL, et al. Understanding the mechanism(s) of mosaic trisomy 21 by using DNA polymorphism analysis. Am f Hum Genet 1994;54: 473-81. 